\title{
Functional Meta-programming for Parallel Skeletons
}

\author{
Jocelyn Serot $^{1}$ and Joel Falcou ${ }^{2}$ \\ ${ }^{1}$ LASMEA, UMR 66O2 CNRS/U. Blaise Pascal, Campus des Cézeaux \\ F-63177 Aubière, France \\ ${ }^{2}$ IEF, Université Paris-Sud, F-91405 Orsay Cedex, France \\ Jocelyn.Serot@lasmea.univ-bpclermont.fr, falcou@ief.u-psud.fr
}

\begin{abstract}
We describe the implementation in MetaOcAmL of a small domain specific language for skeleton-based parallel programming. We show how the meta-programming facilities offered by this language make it possible to virtually eliminate the run-time overhead for the resulting programs, compared to a hand-crafted, low-level implementation.
\end{abstract}

Keywords: Parallel programming, skeletons, meta-programming.

\section{Introduction}

The now well-known concept of skeletons [1] has been proposed as a solution to the problems raised by low-level parallel programming using message-passing libraries such as MPI. With skeletons, parallel programming boils down to choosing, instantiating and combining high-level, generic constructors taken from a predefined library. Skeletons therefore define a small domain specific language (DSL) providing the requested level of abstraction, by which parallel programs can be built without having to deal with low-level implementation details. Several practical realisations of the skeleton concept have been proposed 2476614 . These realisations essentially differ in the way the related DSL is implemented. In practice, one has to define 1) the syntax and semantics of this DSL 2) the transformation rules used for turning the DSL-level expressions into a low-level implementation (using MPIcalls for instance).

For most cited systems, the DSL is implemented as a library within a classical, sequential host language $(\mathrm{C}, \mathrm{C}++, \mathrm{Caml})$. This has two advantages. First, this spares the implementor from having to write a dedicated lexical analyser and parser. Second, it greatly eases the interfacing to sequential functions (either because these functions are written within the host language or because its foreign function interface can be directly reused). The price to pay, on the other hand, is a significant run-time overhead for the resulting code, compared with a hand-crafted implementation of the same application using low-level MPI calls.

In this paper, we explain how partial evaluation and meta-programming techniques - which have already been successfully applied in other contexts - can be used to solve the aforementioned problem. More precisely, we describe the implementation, in METAOCAML, of a small DSL allowing 
- high-level specification of parallel programs as a combination of skeletons,

- automatic generation of the underlying, equivalent low-level parallel code as a set of sequential communicating processes,

- execution of this code on a cluster architecture supporting the MPIlibrary.

The paper is organised as follows. Section 2 briefly recalls what is metaprogramming and how it is supported within the METAOCAML programming language. In Section 3 we explain why this technique is interesting in the context of message-based parallel programming. In Section 4 we introduce a DSL dedicated to skeleton-based parallel programming, giving its syntax and semantics. We then describe how this (formal) semantics can be used to derive an implementation in MetaOcaml. Preliminary experimental results are given in Section 6, Related work is discussed in Section 7. We conclude by summarizing our contributions and giving hints for further work.

\section{Meta-programming with MetaOcaml}

Meta-programming is a technique allowing programs (so-called meta-programs) to analyse, transform and generate other programs (so-called object programs). MetaOcamL [15] is an extension of the OCAML language offering support for meta-programming thanks to three special value constructors, named brackets, escape and run, and a special type constructor, named code.

- Brackets $(.<>$.) enclose an object program part :

\# let $\mathrm{a}=.<1+2>$.; ;

val a : int code $=.\langle(1+2)\rangle$.

The expression $1+2$ is not evaluated and the meta-variable a now holds its representation. The corresponding code fragment can now be inserted in other programs or compiled and executed.

- The escape operator $\left(.{ }^{\sim}\right)$ inserts an object program part into a larger part :

\#let $\mathrm{b}=.<3 *$. $\mathrm{a}>$.; ;

val $\mathrm{b}$ : int code $=.\langle(3 *(1+2))\rangle$.

- The run operator (.!) evaluates (compile and run) an object program :

\#let $c=. ! b ;$

val $c$ : int $=9$

\section{Meta-programming Applied to Parallel Programming}

In order to see how meta-programming "à la METAOCAML" can be be interesting in the context of parallel programming, let's consider the (otherwise meaningless) program depicted in Figure 1 1 a. In this program, each process with an even rank sends a value (computed by the $f$ function) to the process with a rank 


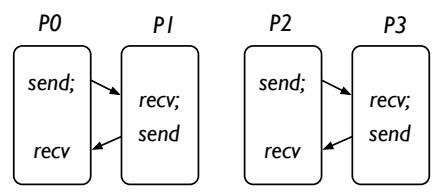

$-a-$

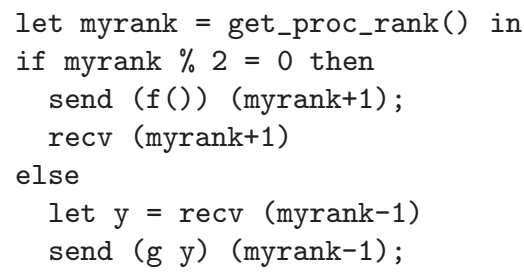

$-b-$

Fig. 1. Example 1

immediately above and then waits for a return value from the same process. Each process with an odd rank waits for a value from the process with a rank immediately below, applies a function $g$ to this value and sends the result to this process.

On almost all cluster architectures, the execution model is SPMD (Single Program Multiple Data). Written with point-to-point communication primitives like send and recv, the corresponding program would therefore look like in Figure $1 \mathrm{t} \mathrm{d} 1$.

In this program, the communication pattern is fixed. The code to be executed by each process is then known as soon as its rank is known. We can take advantage of this to rewrite it in the following manner with METAOCAML:

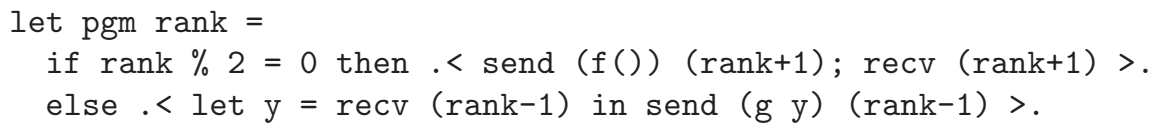

let myrank = get_proc_rank () in

\section{.! (pgm myrank)}

Now, the code to be executed is dynamically generated by each process and then executed. This technique is powerful because it allows this code to be specialised - and optimized - according to parameters which are only known at launch time 2 . It could be objected that this incurs a runtime overhead but this overhead is only paid once, at launch time, and can therefore completely be covered by the gain in performance resulting from executing an optimized code. In the sequel, we will apply this idea to obtain an efficient implementation of a DSL for skeleton-based parallel programming.

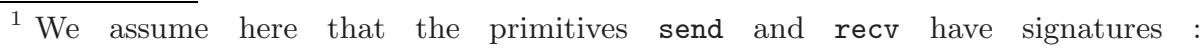
val send: 'a $\rightarrow$ pid $\rightarrow$ unit and val recv: pid $\rightarrow$ 'a and that the primitive get_proc_rank returns, at runtime, the rank of the executing process.

2 This should be contrasted to other languages supporting compile-time metaprogramming, such as $\mathrm{C}++$ or Template Haskell 9 .
} 


\section{A DSL for Skeleton-Based Parallel Programming}

Syntax. The abstract syntax of this DSL - let's call it SKL- is defined below:

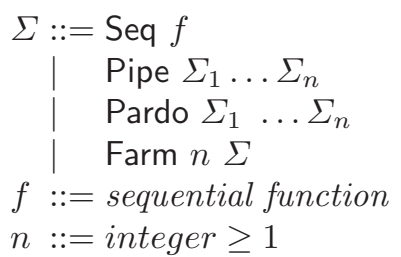

The skeleton set is classical. Intuitively, Seq encapsulates sequential userdefined functions in such a way they can be used as parameters to other skeletons; Pipe and Farm are the usual data-parallel skeletons. Pipe handles situations in which computations are performed in stages. Farm uses a master/workers scheme to implement dynamic load-balancing strategies. Pardo models parallel, independent computations, where $n$ distinct tasks are run on $n$ distinct processors.

Semantics. Due to space limitations, we only give here the semantics of a subset of the language, namely the one built upon the Pipe and Pardo skeletons. This simplified version should suffice to convey the general ideas and show that the implementation sketched in Section 5 is well-founded. The full version of the semantics can be found in [12].

The implementation model of SKL is CSP-based. A parallel program is described as a process network, i.e. a set of processes communicating by channels and executing each a sequence of instructions.

Formally, a process network $(\mathrm{PN})$ is a triple $\pi=\langle P, I, O\rangle$ where

- $P$ is a set of labeled processes, i.e. pairs $(p i d, \sigma)$ where pid is a (unique) process id and $\sigma$ a triple containing: a list of predecessors (pids of processes $p$ for which a communication channel exists from $p$ to the current process), a list of successors (pids of processes $p$ for which a communication channel exists from the current process to $p$ ) and a descriptor $\Delta$. We note $\mathcal{L}(\pi)$ the set of pids of a process network $\pi$. For a process $p$, its predecessors, successors and descriptor will be denoted $\mathcal{I}(p), \mathcal{O}(p)$ et $\delta(p)$ respectively.

$-I(\pi) \subseteq \mathcal{L}(\pi)$ denotes the set of source processes for the network $\pi$ (i.e. the set of processes $p$ for which $\mathcal{I}(p)=\emptyset$ )

$-O(\pi) \subseteq \mathcal{L}(\pi)$ denotes the set of $\operatorname{sink}$ processes for the network $\pi$ (i.e. the set of processes $p$ for which $\mathcal{O}(p)=\emptyset)$

The process descriptor $\Delta$ is a sequence of (abstract) instructions, implicitly iterated (processes never terminate). Instructions use implicit addressing, with each process holding two variables named $v i$, vo. A (simplified) instruction set is given below. In the subsequent explanations, $p$ designates the process executing the instruction.

$$
\text { instr }::=\text { SendTo } \mid \text { RecvFrom | CallFn fid }
$$

The SendTo instruction sends the contents of variable vo to the process whose pid is given in $\mathcal{O}(p)$. The RecvFrom instruction receives data from the process 
whose pid is given in $\mathcal{O}(p)$ and puts it in the variable vi. The CallFn instruction performs a computation by calling a sequential function. This function takes one argument (in vi) and produces one result (in vo).

The transformation of the skeleton tree describing the application into a process network is formalized by means of a set of production rules derived from a basic process algebra.

The following notation will be used. If $\mathcal{E}$ is a set, we denote by $\mathcal{E}\left[e \leftarrow e^{\prime}\right]$ the set obtained by replacing $e$ by $e^{\prime}$ (assuming $\mathcal{E}\left[e \leftarrow e^{\prime}\right]=\mathcal{E}$ if $e \notin \mathcal{E}$ ). This notation is left-associative: $\mathcal{E}\left[e \leftarrow e^{\prime}\right]\left[f \leftarrow f^{\prime}\right]$ means $\left(\mathcal{E}\left[e \leftarrow e^{\prime}\right]\right)\left[f \leftarrow f^{\prime}\right]$. If $e_{1}, \ldots, e_{m}$ is an indexed subset of $\mathcal{E}$ and $\phi: \mathcal{E} \rightarrow \mathcal{E}$ a function, we will note $\mathcal{E}\left[e_{i} \leftarrow \phi\left(e_{i}\right)\right]_{i=1 . . m}$ the set $\left(\ldots\left(\left(\mathcal{E}\left[e_{1} \leftarrow \phi\left(e_{1}\right)\right]\right)\left[e_{2} \leftarrow \phi\left(e_{2}\right)\right]\right) \ldots\right)\left[e_{m} \leftarrow \phi\left(e_{m}\right)\right]$. Except when explicitly indicated, we will note $I\left(\pi_{k}\right)=\left\{i_{k}^{1}, \ldots, i_{k}^{m}\right\}$ and $O\left(\pi_{k}\right)=\left\{o_{k}^{1}, \ldots, o_{k}^{n}\right\}$. For concision, the lists $\mathcal{I}\left(o_{k}^{j}\right)$ et $\mathcal{O}\left(i_{k}^{j}\right)$ will be noted $s_{k}^{j}$ et $d_{k}^{j}$ respectively. For lists, we define the a concatenation operation ++ as usual : if $l_{1}=\left[e_{1}^{1}, \ldots, e_{1}^{m}\right]$ and $l_{2}=\left[e_{2}^{1}, \ldots, e_{2}^{n}\right]$ then $l_{1}++l_{2}=\left[e_{1}^{1}, \ldots, e_{1}^{m}, e_{2}^{1}, \ldots ; e_{2}^{n}\right]$. The empty list is noted []. The length of list $l$ (resp. cardinal of a set $l$ ) is noted $|l|$.

The $\lceil$.$\rceil operator creates a process network containing a single process from a$ process descriptor, using the function $\mathrm{New}()$ to provide "fresh" process ids :

$$
\frac{\delta \in \Delta \quad l=\operatorname{New}()}{\lceil\delta\rceil=\langle\{(l,\langle[],[], \delta\rangle)\},\{l\},\{l\}\rangle}
$$

The • operation "serializes" two process networks, by connecting outputs of the first to the inputs of the second :

$$
\frac{\pi_{i}=\left\langle P_{i}, I_{i}, O_{i}\right\rangle(i=1,2) \quad\left|O_{1}\right|=\left|I_{2}\right|=m}{\frac{\pi_{1} \bullet \pi_{2}=\left\langle\left(P_{1} \cup P_{2}\right)\left[\left(o_{1}^{j}, \sigma\right) \leftarrow \phi_{d}\left(\left(o_{1}^{j}, \sigma\right), i_{2}^{j}\right)\right]_{j=1 \ldots m}\left[\left(i_{2}^{j}, \sigma\right) \leftarrow \phi_{s}\left(\left(i_{2}^{j}, \sigma\right), o_{1}^{j}\right)\right]_{j=1 \ldots m}\right.}{\left.I_{1}, O_{2}\right\rangle}}
$$

(SERIal)

This rule uses two auxiliary functions $\phi_{s}$ and $\phi_{d}$ defined as follows :

$$
\begin{aligned}
& \phi_{s}\left((p,\langle s, d, \delta\rangle), p^{\prime}\right)=\left(p,\left\langle\left[p^{\prime}\right]++s, d,[\text { RecvFrom }]++\delta\right\rangle\right) \\
& \phi_{d}\left((p,\langle s, d, \delta\rangle), p^{\prime}\right)=\left(p,\left\langle s, d++\left[p^{\prime}\right], \delta++[\text { SendTo }]\right\rangle\right)
\end{aligned}
$$

The function $\phi_{s}$ (resp. $\phi_{d}$ ) adds a process $p^{\prime}$ as a predecessor (resp. successor) to process $p$ and updates accordingly its instruction list. This involves prepending (resp. appending) a RecvFrom (resp. SendTo) instruction) to this instruction list.

The $\|$ operation puts two process networks in parallel, merging their inputs and outputs respectively.

$$
\frac{\pi_{i}=\left\langle P_{i}, I_{i}, O_{i}\right\rangle(i=1,2)}{\pi_{1} \| \pi_{2}=\left\langle P_{1} \cup P_{2}, I_{1} \cup I_{2}, O_{1} \cup O_{2}\right\rangle}
$$


Skeletons can now be defined in terms of the operations defined above, using the following conversion function $\mathcal{C}$ :

$$
\begin{aligned}
\mathcal{C}[[\text { Seq } f]] & =\lceil f\rceil \\
\mathcal{C}\left[\left[\text { Pipe } \Sigma_{1} \ldots \Sigma_{n}\right]\right] & =\mathcal{C}\left[\left[\Sigma_{1}\right]\right] \bullet \ldots \bullet \mathcal{C}\left[\left[\Sigma_{n}\right]\right] \\
\mathcal{C}\left[\left[\text { Pardo } \Sigma_{1} \ldots \Sigma_{n}\right]\right] & =\mathcal{C}\left[\left[\Sigma_{1}\right]\right]\|\ldots\| \mathcal{C}\left[\left[\Sigma_{n}\right]\right]
\end{aligned}
$$

\section{Implementation}

Embedding the SkL language within MetaOCAML is done by providing an interpretation function (run) which converts an SKL program into distinct pieces of residual code on each processor. This conversion is performed in two steps: First, the process network representation of the program is obtained from the tree-based one using the production rules defined by the semantics. Second, the residual code is generated by finding, in this representation, the process having the rank of the current process - the one executing the run function - and by converting its sequence of instructions into OCAML code :

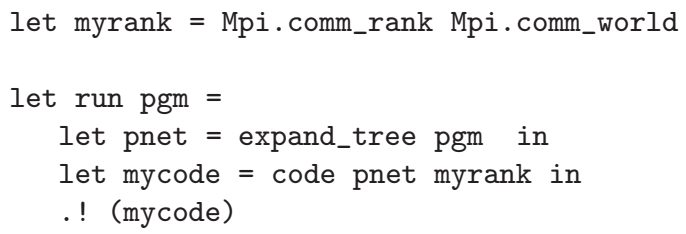

The expand_tree function implements the first step. For example, we have

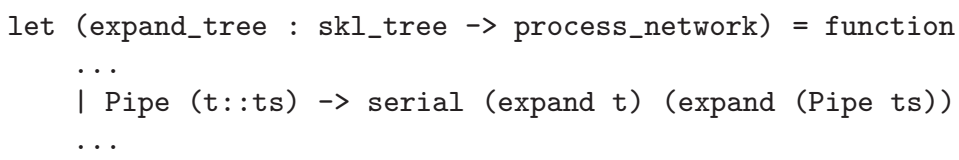

where serial implementes the $\bullet$ operator defined in the previous section.

The code function implements the second step :

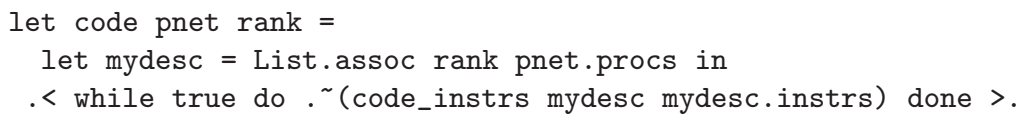

The residual code is producing by traversing the list of macro-instructions attached to the current process:

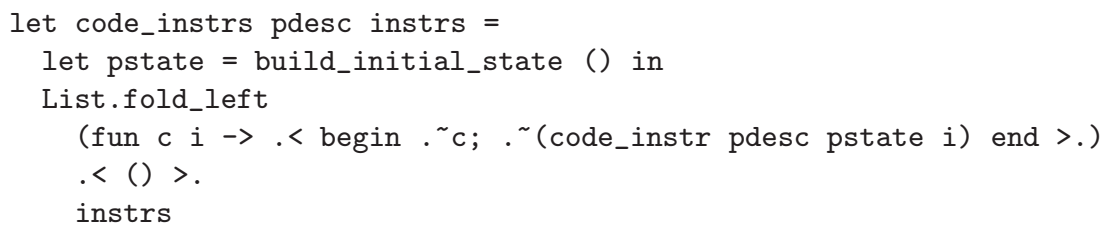


The pstate variable will hold the (dynamic) process state. This state includes the last input and output values (iv and ov resp.), the pid of the sending process for the last receive instruction and the list of idle workers (for farm masters 3

Translation of macro-instructions into OCAML code is performed by the code_instr function. The use of implicit addressing greatly eases this translation, since arguments and results are read (resp. written) directly from (resp. to) the process state (no variable environment is needed). For example, we have:

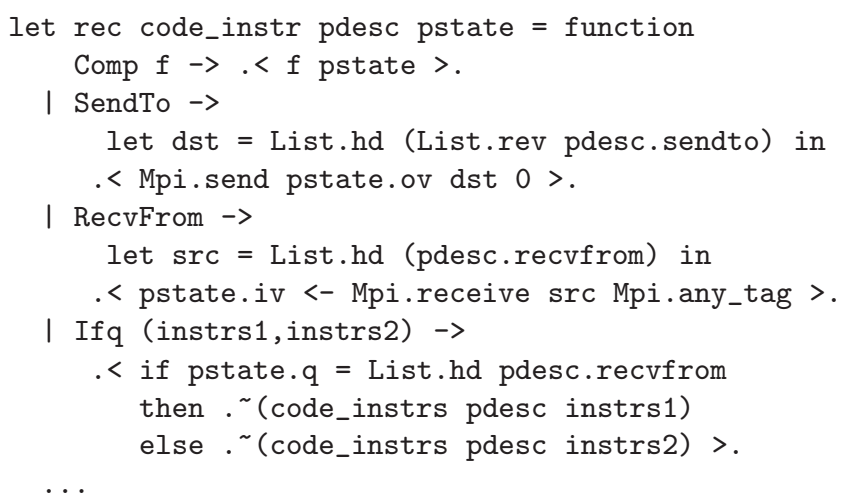

A CallFn $\mathrm{f}$ instruction generates a call to the sequential function $f$. SendTo, and RecvFrom instructions are translated into direct calls to MPIprimitives (we use here the OCAMLMPI 13 library, v1.0.1). For the conditional instruction Ifq , the escape operator of METAOCAML allows the insertion of the selected branch in the residual codet

Example 1. Consider a simple SKL program describing a three-stage pipeline: data are produced at stage 1 , processed at stage 2 , and results are consumed at stage 3. In METAOCAML, this program will be written as:

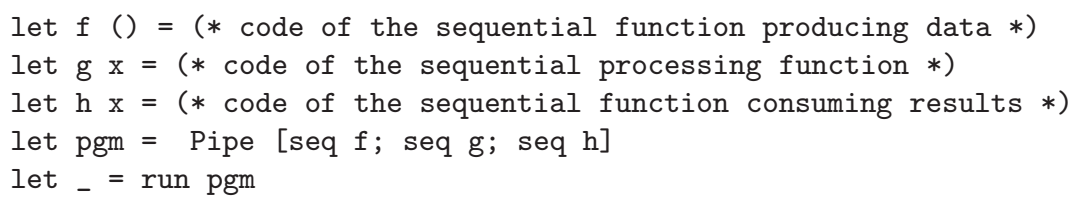

The residual code produced on each processor is as follows:

\begin{tabular}{|c|l|}
\hline PID & Code \\
\hline 2 & while true do f s; Mpi.send s.ov 10 done \\
\hline 1 & $\begin{array}{l}\text { while true do } \\
\text { s.iv <- Mpi.receive 2 0; g s; Mpi.send s.ov } 00 \\
\text { done }\end{array}$ \\
\hline 0 & while true do s.iv <- Mpi.receive 10 ; h s done \\
\hline
\end{tabular}

${ }^{3}$ Our approach therefore explicitly relies on cross-stage persistence, i.e. the possibility of defining of variable - like pstate - at stage $i$ and using it at stage $i+1$.

${ }^{4}$ The RecvFrom and Ifq instructions are used by processes involved in the farm skeleton; they were therefore not introduced in simplified semantics described in the previous section. 
Example 2. Consider now a three-stage pipeline, in which the second stage is a farm involving a master and two workers :

let $\mathrm{pgm}=\operatorname{Pipe}[$ seq $\mathrm{f} ; \operatorname{Farm}(2$, seq $\mathrm{g}) ;$ seq $\mathrm{h}]$

let _ $=$ run pgm

The residual code produced on each processor is now :

\begin{tabular}{|c|l|}
\hline PID & Code \\
\hline 4 & while true do f s; Mpi.send s.ov 30 done \\
\hline 3 & while true do \\
& let r, q, - = Mpi.receive_status 00 in \\
& s.ov <- r; s.q <- q; \\
& if s.q = List.hd pd.recvfrom then begin \\
& let q = get_idle_worker iws in Mpi.send s.ov q 0 end \\
& else begin \\
& update_workers s.q s.iws; Mpi.send <- s.ov 00 end \\
& done \\
\hline 2 & while true s.iv <- Mpi.receive $30 ;$ f s; Mpi.send s.ov 30 done \\
\hline 1 & while true s.iv <- Mpi.receive $30 ; f$ s; Mpi.send s.ov 30 done \\
\hline 0 & while true s.iv <- Mpi.receive $30 ;$ s done \\
\hline
\end{tabular}

In both cases, the code is very similar to the one that an experienced MPIprogrammer would have written.

\section{Results}

We have assessed the impact of this implementation technique by measuring the overhead $\rho$ on the completion time over hand-written OCAML + OCAMLMPI code for both single skeleton application and when skeletons are nested at arbitrary level. For single skeleton tests, we observe the effect of two parameters: $\tau$, the execution time of the inner sequential function and $N$, the "size" of the skeleton (number of stages for PIPELINE, number of workers for FARM. The test case for nesting skeletons involved nesting $P$ FARM skeletons, each having $\omega$ workers. Results were obtained on a PowerPC G5 cluster with 30 processors and for $N=2-30$ and $\tau=1 \mathrm{~ms}, 10 \mathrm{~ms}, 100 \mathrm{~ms}, 1 \mathrm{~s}$.

For PIPELINE, $\rho$ stays under $2 \%$. For FARM, $\rho$ is no greater than $3 \%$ and becomes negligible for $N>8$ or $\tau>10 \mathrm{~ms}$. For the nesting test, worst case is obtained with $P=4$ and $\omega=2$. In this case, $\rho$ decreases from $7 \%$ to $3 \%$ when $\tau$ increases from $10^{-3} s$ to $1 s$.

These results are significantly better than those obtained with skeleton-based parallel programming systems which do not exploit meta-programming. In the implementation described in [10, for instance, where skeletons are implemented as simple higher-order functions dynamically scheduling MPI calls, the overhead was between 10 and $20 \%$. For the last version of the SKIPPER system [11, in which skeletons were translated into macro data-flow graphs executed by a distributed interpreter, this overhead could reach reach 100\%. For implementations 
relying on object-oriented techniques, such as that described by Kuchen [4, the reported overhead (compared to $\mathrm{C}+\mathrm{MPI}$ code in this case) is between 20 and $120 \%$.

\section{Related Work}

The idea of exploiting meta-programming techniques to implement a DSL is definitely not new. Reviews of related issues can be found for example in [8] (in the context of functional programming) or in [5] (in the context of parallel programming).

In 12 we have already described a skeleton-based parallel programming system exploiting the static meta-programming facilities offered by the $\mathrm{C}++$ template mechanism. The work described here is based upon the same semantics and process network model but differs in the host language and in the fact that the residual code is now produced at runtime, using the dynamic code generation facilities of METAOCAML.

This idea of exploiting the dynamic meta and multi-stage programming facilities offered by a language such METAOCAML to implement a DSL seems to have been followed only by Herrmann in [3]. The system which he describes is very similar to ours but nevertheless differs in several points. First, skeletons are not part of the DSL per se. Instead, they can be defined in terms of the constructs introduced by this DSL. Second, skeletons for which the scheduling of communications cannot be computed at compile-time - such as Farm - are not supported. Third, the semantics of the DSL is not defined formally but is instead directly encoded in the METAOCAML interpreter (there's no explicit intermediate representation such as a process network).

\section{Conclusion}

We have shown in this paper how the meta-programming facilities offered by a language such as MetaOCAML can be used to implement efficiently a DSL dedicated to skeleton-based parallel programming. The (runtime) specialisation of code for each distinct processor makes it possible to eliminate the overhead observed in other skeleton-based approaches to parallel programming. The resulting system offers a high level of abstraction and performances on the par with hand-crafted MPIcode.

The prototype described here is however more a proof-of-concept than a fullfledged programming system and several issues could be further investigated. In particular, the fact that the residual code is generated at run-time on each processor makes it possible to customize and optimize this code according to the actual profile of this processor. This possibility is clearly "under-used" in our implementation, in which only the processor rank is taken into account. More sophisticated strategies could easily be developed, taking into account the processor computing capabilities, actual workload, etc. This could prove very useful for handling heterogeneous architectures, for instance. 


\section{References}

1. Cole, M.: Algorithmic skeletons, ch.13. Research Directions in Parallel Functional Programming. Springer, Heidelberg (1999)

2. Bacci, B., Danelutto, M., Orlando, S., Pelagatti, S., Vanneschi, M.: P3L: A Structured High Level Programming Language And Its Structured Support. Concurrency: Practice and Experience, 225-255 (1995)

3. Herrmann, C.: Generating message-passing programs from abstract specifications by partial evaluation. Parallel Processing Letters 15(3), 305-320 (2005)

4. Kuchen, H.: A skeleton library. In: Monien, B., Feldmann, R.L. (eds.) Euro-Par 2002. LNCS, vol. 2400, pp. 620-629. Springer, Heidelberg (2002)

5. Lengauer, C., Batory, D., Consel, C., Odersky, M. (eds.): Domain-Specific Program Generation. LNCS, vol. 3016. Springer, Heidelberg (2004)

6. Michaelson, G., Scaife, N., Horiguchi, S.: Parallel Standard ML with Skeletons. Scalable Computing, Practise and Experience 6(4) (2006)

7. Sérot, J., Ginhac, D.: Skeletons for parallel image processing: an overview of the SKiPPER project. Parallel Computing 28(12), 1785-1808 (2002)

8. Sheard, T.: Accomplishments and research challenges in meta-programming. In: Taha, W. (ed.) SAIG 2001. LNCS, vol. 2196, pp. 2-44. Springer, Heidelberg (2001)

9. Sheard, T., Peyton-Jones, S.: Template metaprogramming for Haskell. In: Chakravarty, M.M.T. (ed.) ACM SIGPLAN Haskell Workshop 2002, pp. 1-16. ACM Press, New York (2002)

10. Sérot, J.: Embodying parallel functional skeletons: an experimental implementation on top of MPI. In: Lengauer, C., Griebl, M., Gorlatch, S. (eds.) Euro-Par 1997. LNCS, vol. 1300, pp. 629-633. Springer, Heidelberg (1997)

11. Sérot, J.: Tagged-token data-flow for skeletons. Parallel Processing Letters 11(4), 377-392 (2002)

12. Falcou, J., Sérot, J.: Formal semantics applied to the implementation of a skeletonbased parallel programming library. In: Proceedings of the International Conference ParCo 2007, Aachen. Advances in Parallel Computing, vol. 15, IOS Press, Amsterdam (2008)

13. OcamlMPI, http://caml.inria.fr/cgi-bin/hump.en.cgi?contrib=401

14. OcamlP3L, http://ocamlp3l.inria.fr

15. MetaOcaml, http://www.metaocaml.com 